\title{
Künstliche Intelligenz - kein Weg führt daran vorbei!
} |ELSAAHAN|D|

Helfen Sie mit, die ethischen und sozialen Fragen der Zukunft der Medizin zu definieren!

In den kommenden Jahren ist davon auszugehen, dass sich durch die zunehmende Digitalisierung in der Medizin der klinische Alltag deutlich verändern wird. Neue Technologien, wie Künstliche Intelligenz (KI), können medizinischem Fachpersonal dabei helfen, ihren Arbeitsalltag effektiver zu gestalten. Trotz großer Fortschritte in der KI-unterstützten Diagnostik wurden soziale und ethische Konsequenzen - obwohl essenziell für die Implementierung in der Klinik - kaum untersucht.

Ansinnen der BMBF-Fördermaßnahme „Forschung zu ethischen, rechtlichen und sozialen Aspekten (ELSA) der Digitalisierung, von
Big Data und Künstlicher Intelligenz in der Gesundheitsforschung und -versorgung" ist es, die Auswirkungen der neuen Technologien auf Wissenschaft und Gesellschaft zu untersuchen und auf einen gesellschaftlich akzeptierten und verantworteten Rahmen für ihren Einsatz hinzuwirken.

Gegenstand des vom BMBF in diesem $\mathrm{Zu}$ sammenhang geförderten Verbundprojekt ELSA-AID der RWTH, Institut für Angewandte Ethik, der Uniklinik RWTH Aachen, Institut für Experimentelle Molekulare Bildgebung und des Fraunhofer MEVIS-Instituts ist eine sensible Analyse ethischer, sozialer und beruflicher Fragen der KI-gestützten Diagnostik im Rahmen der Präzisionsmedizin.

Um ein allgemeines Stimmungsbild von Ärztinnen und Ärzten zum Thema KI in der Medizin zu erstellen, das einen grundlegenden Baustein für die Erstellung eines Konzepts zur Implementierung von $\mathrm{KI}$ in der Präzisionsmedizin setzen wird, hat ELSA-
AID eine Umfrage erstellt. Diese richtet sich an alle DRG-Mitglieder.

Wir würden uns sehr freuen, wenn auch Sie an der 10-minütigen anonymisierten Umfrage des Verbundprojekts ELSA-AID, das von der DRG unterstützt wird, teilnehmen. Gerne können Sie die Umfrage auch an Ihre Kolleginnen und Kollegen weiterleiten. Vielen Dank!

Verantwortliche für die Umfrage:

Helen Heinrichs M.Sc., Institut für Experimentelle Molekulare Bildgebung, Uniklinik RWTH Aachen

Projektleitung:

Univ.-Prof. Dr. Saskia Nagel, Prof. Dr. Horst Hahn, Univ.-Prof. Dr. med. Fabian Kiessling

Den Link zur Umfrage finden Sie unter: www.drg.de > Aktuelles > Künstliche Intelligenz - kein Weg führt daran vorbei! 